\title{
Compact rf polarizer and its application to pulse compression systems
}

\author{
Matthew Franzi, Juwen Wang, Valery Dolgashev, and Sami Tantawi \\ SLAC National Accelerator Laboratory, 2575 Sand Hill Road, Menlo Park, California 94025, USA
}

(Received 25 January 2016; published 17 June 2016)

\begin{abstract}
We present a novel method of reducing the footprint and increasing the efficiency of the modern multiMW rf pulse compressor. This system utilizes a high power rf polarizer to couple two circular waveguide modes in quadrature to a single resonant cavity in order to replicate the response of a traditional two cavity configuration using a 4-port hybrid. The $11.424 \mathrm{GHz}$, high-Q, spherical cavity has a $5.875 \mathrm{~cm}$ radius and is fed by the circularly polarized signal to simultaneously excite the degenerate $T E_{114}$ modes. The overcoupled spherical cavity has a $Q_{0}$ of $9.4 \times 10^{4}$ and coupling factor $(\beta)$ of 7.69 thus providing a loaded quality factor $Q_{L}$ of $1.06 \times 10^{4}$ with a fill time of $150 \mathrm{~ns}$. Cold tests of the polarizer demonstrated good agreement with the numerical design, showing transmission of $-0.05 \mathrm{~dB}$ and reflection back to the input rectangular WR 90 waveguide less than $-40 \mathrm{~dB}$ over a $100 \mathrm{MHz}$ bandwidth. This novel rf pulse compressor was tested at SLAC using XL-4 Klystron that provided rf power up to $32 \mathrm{MW}$ and generated peak output power of $205 \mathrm{MW}$ and an average of $135 \mathrm{MW}$ over the discharged signal. A general network analysis of the polarizer is discussed as well as the design and high power test of the rf pulse compressor.
\end{abstract}

DOI: 10.1103/PhysRevAccelBeams.19.062002

\section{INTRODUCTION}

High power microwave (HPM) devices are used in multiple scientific and industrial applications including particle accelerators, communication, and defense technology $[1,2]$. Large scale rf particle accelerators in particular require high power microwave pulses, on the order of hundreds of megawatts, as well as precise control of signal's amplitude and phase. The stringent demands of accelerator applications are translated directly to the requirements for rf source parameters thus increasing the cost and complexity of the devices. Radio frequency pulse compression is often utilized to alleviate this cost by transforming a low power, long pulse, input signal to a higher power, short pulse, output. This process reduces the required power output of the source allowing for the implementation of a cheaper, more efficient, device. Radio frequency pulse compression is a mature technology which can be accomplished through several different methods [3-5]. This article focuses on the SLED-I configuration currently employed by the SLAC S-band linac.

The SLED-I system currently requires the use of two over-coupled cylindrical resonant cavities and a 4-port $3 \mathrm{~dB}$ hybrid in order to isolate the source from the reflected $\mathrm{rf}$ signal from cavities. The novel rf pulse compressor presented in this article emulates this mechanism for isolation by using a single resonant cavity excited by circularly polarized mode via a novel 3-port, 4-mode, rf

Published by the American Physical Society under the terms of the Creative Commons Attribution 3.0 License. Further distribution of this work must maintain attribution to the author(s) and the published article's title, journal citation, and DOI. polarizer [6,7]. This new configuration reduces system footprint by $50 \%$ reduction in the devices overall footprint, reducing both complexity as well as ohmic losses during operation.

Radio frequency polarizers are a highly versatile class of devices that can be utilized in a number of applications including phase-shifters, duplexer's, loads, and hybrid couplers [8,9]. Existing embodiments of the polarizer, however, require the use of complicated structures such as irises, septums, or corrugations, in order to properly excite the circularly polarized output signal [9-11]. These features can increase electromagnetic fields and thus significantly reduce the devices power handling capabilities due to heating and breakdown. The rf polarizer presented here is a compact device that utilizes only smooth, rounded, surfaces to minimize this enhancement while maintaining the inherent high power handling capabilities of an overmoded system [12]. This article addresses both the network analysis of the polarizer and the device's role in improving rf pulse compressor technology at SLAC National Accelerator Laboratory.

\section{RF POLARIZER}

The polarizer in Fig. 1 is comprised of two symmetric rectangular waveguide ports which feed a cylindrical transmission line via an overmoded rectangular waveguide. The overmoded portion of the $\mathrm{rf}$ network is designed to support the simultaneous propagation of the $T E_{10}^{\square}$ and $T E_{20}^{\square}$ mode which couple to two $T E_{11}^{O}$ cylindrical modes in quadrature. Proper coupling to the $T E_{11}^{O}$ modes is obtained by precise tuning of a shorted cylindrical waveguide section that extends from the base of the rectangular waveguide. Additionally, E-plane fillets on the back wall 


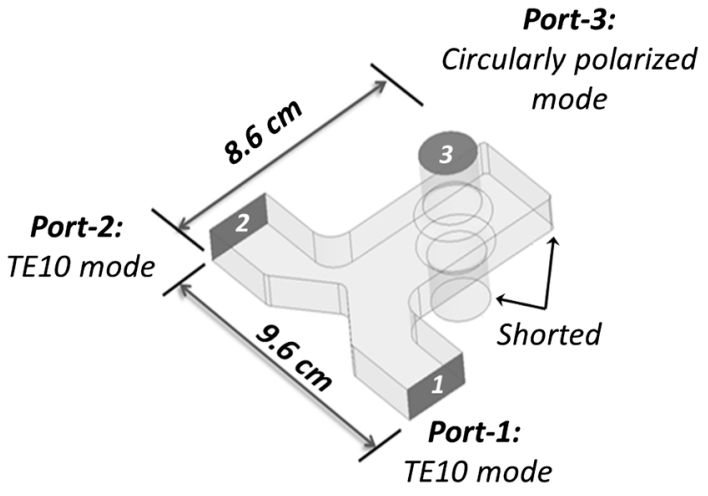

FIG. 1. 3D rendering of an $11.424 \mathrm{GHz}$, x-band, rf polarizer with physical ports and waveguide types labeled accordingly.

act to asymmetrically adjust the rectangular boundary for both the $T E_{10}^{\square}$ and $T E_{20}^{\square}$ modes in order to accommodate their dissimilar propagation constants. Optimization of these parameters, and the respective length of the rectangular waveguide section, enables both excitation of the $T E_{11}^{O}$ modes in quadrature as well as nearly perfect isolation between ports-1 and 2. The superposition of the orthogonal modes within the circular waveguide, generates a right hand circularly polarized (RHCP) or left hand circularly polarized (LHCP) "like" wave. It should be noted that, in this analysis, rectangular waveguide modes will be denoted as $T E^{\square}$ while circular waveguide modes are described as $T E^{O}$.

\section{A. Microwave network analysis}

A network analysis of this unique device is performed by first dividing the system into three separate components denoted as network (A-C). Network-A, shown in Fig. 2, is a 4-port circuit which contains two rectangular waveguide (single-mode) input ports and the portion of overmoded rectangular waveguide (2-modes). Network-B, is a 6-port circuit consisting of two overmoded rectangular waveguide boundaries (2-modes each) on either end of a single cylindrical waveguide boundary (2-modes). The remaining portion, network-C, is a shorted length of the rectangular waveguide (2-modes) that terminates one end of the overmoded rectangular waveguide. The simplified scattering matrices of each sub-network are defined using basic properties of passive microwave networks and then cascaded together to evaluate the parameters of the full device. All internal ports in this analysis, which will be removed though cascading, are referenced by number, subnetwork, and mode (ie. port-2B1 is port-2, network-B, mode-1). External ports, which remain in the final scattering matrix, are enumerated as well as referenced to a give mode of operation (ie. port-3:1 is port-3, mode-1). Higher order modes such as $T E_{30}^{\square}$ are ignored in this analysis as they are cutoff in each section of transmission line. This assumption

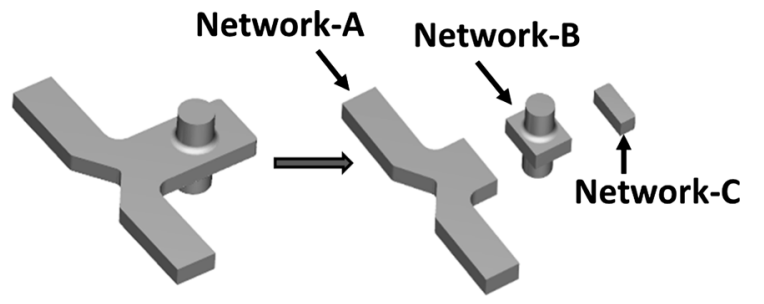

FIG. 2. The rf polarizer divided into each submatrix for cascade analysis.

has been validated in numerical simulations using a finite element model that included higher order terms.

\section{Network $B$}

The polarized output of the cylindrical waveguide in network-B is addressed first in this analysis. This subnetwork, depicted in Fig. 3, contains two ports that will remain external in our system $(3: 1$ and $3: 2)$ and four ports that will be reduced through cascading the remaining subnetworks (1B:1, 1B:2, 2B:1, and 2B:2).

This network is defined by a complex $6 \times 6$ scattering matrix which contains 72 free unknown parameters, but is drawn in such a manner as to exploit the four-fold symmetry of the system. This symmetry allows for an even and odd mode analysis of the subnetwork as it pertains to the $T E_{10}^{\square}$ and $T E_{20}^{\square}$ shown in Fig. 4 respectfully. The even mode is effectively decoupled from the odd by applying a perfect-magnetic boundary along the plane which bisects the H-plane of the rectangular waveguide. The assumed boundary condition will cutoff the $T E_{20}^{\square}$ mode but preserve

\section{Network-B}

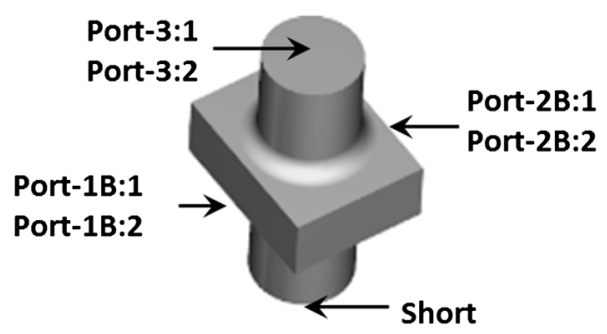

Network-C

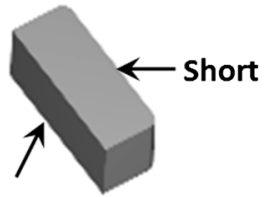

Port-1C:1

Port-1C:2

FIG. 3. Subnetworks A and BC used for the second cascade of the polarizer model with labeled port assignments. 


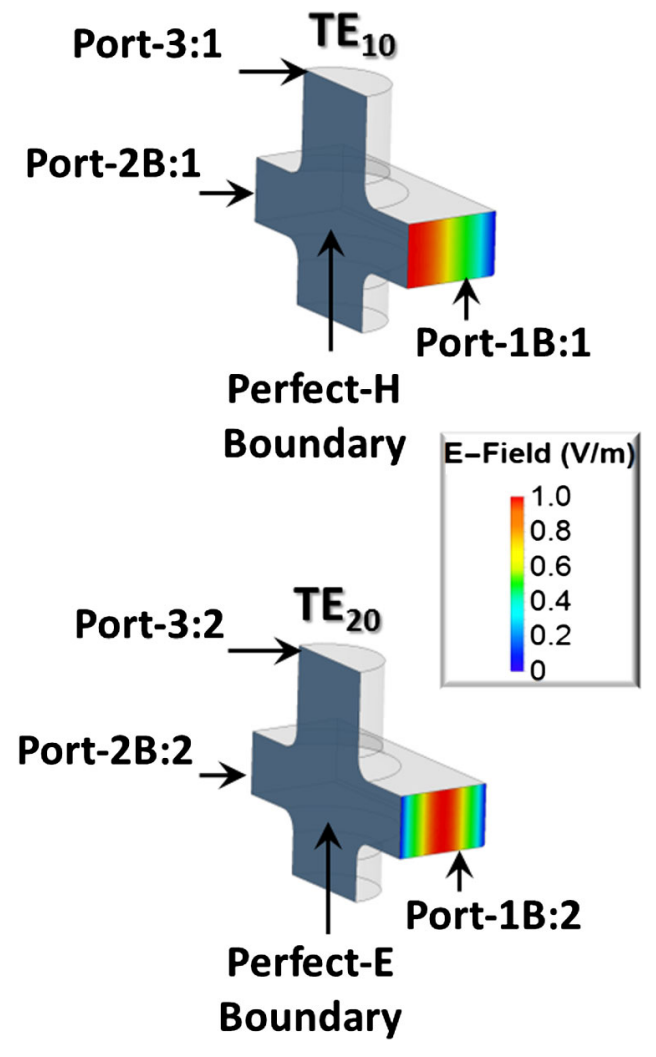

FIG. 4. A cross section view of network-B with the boundary conditions used to isolate the even mode (top) and odd mode (bottom) for this analysis.

the transmission characteristics of the $T E_{10}^{\square}$ mode, effectively reducing the scattering parameters of the subnetwork to a $3 \times 3$ matrix.

All circuits analyzed in this article are considered to be passive and lossless such that the three port unitary subcircuit of network-B cannot be perfectly matched. Knowing a priori that the reflection from the input port cylindrical waveguide must have an absolute magnitude between zero and one, we intuitively assign this term a value $\left(\cos \left(\theta_{1}\right)\right)$. Applying reciprocity to the remaining terms of the scattering matrix simplifies the system to,

$$
S=\left(\begin{array}{ccc}
S_{11} & S_{12} & S_{13} \\
S_{12} & S_{22} & S_{23} \\
S_{13} & S_{23} & \cos \left(\theta_{1}\right)
\end{array}\right) .
$$

The 3-port circuit is also chosen such that perfect symmetry exists from the cylindrical waveguide to either end of the rectangular waveguide. Provided this symmetry, equal coupling to from port- $3: 1$ to ports- $1 \mathrm{~B}: 1$ and $2 \mathrm{~B}: 1$ can be assumed, where $\left(S_{23}=S_{13}=\sin \left(\theta_{1}\right) / \sqrt{2}\right)$. The remaining terms $\left(S_{22}, S_{21}, S_{12}, S_{11}\right)$ are now completely determined by the system of equations resulting in the $3 \times 3$ scatting matrix, [13]

$$
S=\left(\begin{array}{ccc}
\frac{-\cos \left(\theta_{1}\right)-e^{i \phi_{1}}}{2} & \frac{-\cos \left(\theta_{1}\right)+e^{i \phi_{1}}}{2} & \frac{\sin \left(\theta_{1}\right)}{\sqrt{2}} \\
\frac{-\cos \left(\theta_{1}\right)+e^{i \phi_{1}}}{2} & \frac{-\cos \left(\theta_{1}\right)-e^{i \phi_{1}}}{2} & \frac{\sin \left(\theta_{1}\right)}{\sqrt{2}} \\
\frac{\sin \left(\theta_{1}\right)}{\sqrt{2}} & \frac{\sin \left(\theta_{1}\right)}{\sqrt{2}} & \cos \left(\theta_{1}\right)
\end{array}\right)
$$

The term $\left(\phi_{1}\right)$ in Eq. (2) is representative the phase delay of the $T E_{10}^{\square}$ mode passing from one junction of the rectangular waveguide to the other in network-B.

The $T E_{20}^{\square}$ mode can be found using similar techniques except a perfect-electric boundary condition is assumed over the axis of symmetry rather than a perfect-H boundary. The new system will be cutoff to the $T E_{10}^{\square}$ mode and is solved in the same manner as the previous mode but with a different magnitude and phase $\left(\phi_{2}\right.$ and $\left.\theta_{2}\right)$ due the difference in propagation constants. The terms of each $3 \times 3$ scatter matrix are simplified by using the coefficients,

$$
\begin{aligned}
& A_{n}=\frac{-\cos \left(\theta_{n}\right)-e^{i \phi_{n}}}{2} \\
& B_{n}=\frac{-\cos \left(\theta_{n}\right)+e^{i \phi_{n}}}{2} \\
& C_{n}=\frac{\sin \left(\theta_{n}\right)}{\sqrt{2}} \\
& D_{n}=\cos \left(\theta_{n}\right),
\end{aligned}
$$

where the subscript $(n)$ represents the $T E_{10}^{\square}(n=1)$ or $T E_{20}^{\square}$ $(n=2)$ modes. The actual circuit will allow both modes to propagate and are therefore combined to generate the full $6 \times 6$ scatter matrix for network-B,

$$
S=\left(\begin{array}{cccccc}
A_{1} & 0 & B_{1} & 0 & C_{1} & 0 \\
0 & A_{2} & 0 & B_{2} & 0 & C_{2} \\
B_{1} & 0 & A_{1} & 0 & C_{1} & 0 \\
0 & B_{2} & 0 & A_{2} & 0 & C_{2} \\
C_{1} & 0 & C_{1} & 0 & D_{1} & 0 \\
0 & C_{2} & 0 & C_{2} & 0 & D_{2}
\end{array}\right) .
$$

Coupling between the two modes was found to be negligible in finite element analysis and is ignored in this analytic model.

\section{Network C}

The ports $1 \mathrm{C}: 1$ and $1 \mathrm{C}: 2$ of network-C (a short circuit termination shown in Fig. 3) are connected to ports $2 \mathrm{~B}: 1$ and $2 \mathrm{~B}: 2$ of network-B to reduce the complexity of the device. An arbitrary length of overmoded waveguide is included in network-C to compensate for the introduction of the reflection, where $\psi_{1}$ and $\psi_{2}$ are the phase delays for 
each mode and $(\Gamma=-1)$ is the reflection coefficient from the short. Network-B (rearranged based in connectivity),

$$
S=\begin{array}{cccc|cc}
A_{1} & 0 & C_{1} & 0 & \overbrace{B_{1}} & 0 \\
0 & A_{2} & 0 & C_{2} & 0 & B_{2} \\
C_{1} & 0 & D_{1} & 0 & C_{1} & 0 \\
0 & C_{2} & 0 & D_{2} & 0 & C_{2} \\
\hline B_{1} & 0 & C_{1} & 0 & A_{1} & 0 \\
0 & B_{2} & 0 & C_{2} & 0 & A_{2}
\end{array},
$$

$$
\begin{aligned}
S_{B C} & =\left(\begin{array}{ll}
S_{B C 1} & S_{B C 2} \\
S_{B C 3} & S_{B C 4}
\end{array}\right) \\
S_{B C 1} & =S_{B 1}+S_{B 2} \cdot\left(I-S_{C 1} \cdot S_{B 4}\right)^{-1} \cdot S_{C 1} \cdot S_{B 3} \\
S_{B C 2} & =S_{B 2} \cdot\left(I-S_{C 1} \cdot S_{B 4}\right)^{-1} \cdot S_{C 2} \\
S_{B C 3} & =S_{C 3} \cdot\left(I-S_{B 4} \cdot S_{C 1}\right)^{-1} \cdot S_{B 2} \\
S_{B C 4} & =S_{C 4}+S_{C 3} \cdot\left(I-S_{B 4} \cdot S_{C 1}\right)^{-1} \cdot S_{B 4} \cdot S_{C 2} .
\end{aligned}
$$

and network-C,

$$
S_{C}=\left(\begin{array}{cc}
e^{i 2 \psi_{1}} \Gamma & 0 \\
0 & e^{i 2 \psi_{2}} \Gamma
\end{array}\right)=\left(\begin{array}{cc}
Z_{1} & 0 \\
0 & Z_{2}
\end{array}\right),
$$

The lack of external connections in network-C makes terms $S_{C 2}$ through $S_{C 4}$, from Eq. (10), equal to zero and $S_{B C}$ is reduced to the $4 \times 4$ matrix,

$$
S_{B C}=\left(\begin{array}{cccc}
\frac{A_{1}-A_{1}^{2} Z_{1}+B_{1}^{2} Z_{1}}{1-A_{1} Z_{1}} & 0 & \frac{C_{1}\left(A_{1} Z_{1}-B_{1} Z_{1}-1\right)}{A_{1} Z_{1}-1} & 0 \\
0 & \frac{A_{2}-A_{2}^{2} Z_{2}+B_{2}^{2} Z_{2}}{1-A_{2} Z_{2}} & 0 & \frac{C_{2}\left(A_{2} Z_{2}-B_{2} Z_{2}-1\right)}{A_{2} Z_{2}-1} \\
\frac{C_{1}\left(A_{1} Z_{1}-B_{1} Z_{1}-1\right)}{A_{1} Z_{1}-1} & 0 & \frac{D_{1}+C_{1}^{2} Z_{1}-A_{1} D_{1} Z_{1}}{1-A_{1} Z_{1}} & 0 \\
0 & \frac{C_{2}\left(A_{2} Z_{2}-B_{2} Z_{2}-1\right)}{A_{2} Z_{2}-1} & 0 & \frac{D_{2}+C_{2}^{2} Z_{2}-A_{2} D_{2} Z_{2}}{1-A_{2} Z_{2}}
\end{array}\right) .
$$

The matching conditions for network-BC are identified by simultaneously exciting ports-(1:1) and $(1: 2)$, where $V_{\text {out }}=S_{\mathrm{BC}} \cdot V_{\text {in }}, V_{\text {in }}=[1,1,0,0]^{T}$, giving two equations for the normalized mismatch of each mode. This mismatch will be denoted as $\left(\epsilon_{n}\right)$, where $\left(\epsilon_{n}=V_{\text {out }}[n] / V_{\text {in }}[n]=\right.$ $\left.S_{1: n, 1: n}\right)$. The terms $\left(\epsilon_{1}\right)$ and $\left(\epsilon_{2}\right)$ are related to the parameters of the polarizer circuit by plugging in Eqs. (3-6) into Eq. (11) and solving for the phase delay added by network-C for the $T E_{10}^{\square}$ mode,

$\psi_{1}=-i * \ln \left\{-\frac{\sqrt{2 \epsilon_{1}+e^{i \phi_{1}}+\cos \theta_{1}}}{\left.\sqrt{\epsilon_{1} \cos \theta_{1}+e^{i \phi_{1}}\left[\epsilon_{1}+2 \cos \left(\theta_{1}\right)\right.}\right]}\right\}$,

and for the $T E_{20}^{\square}$ mode,

$\psi_{2}=-i * \ln \left\{-\frac{\sqrt{2 \epsilon_{2}+e^{i \phi_{2}}+\cos \theta_{2}}}{\sqrt{\epsilon_{2} \cos \theta_{2}+e^{i \phi_{2}}\left[\epsilon_{2}+2 \cos \left(\theta_{2}\right)\right.}}\right\}$.

The generality of these expressions can be alleviated by assuming a fixed relation between the network parameters of each mode. Here, we assume $\left(\theta_{1}=\theta_{2}, \phi_{1}=\phi_{2}, \epsilon_{1}=\epsilon_{2}\right)$ such that the analysis of modes (1) and (2) are equivalent and the subscript (n) can be dropped. These assumptions may be physically realized by the inclusion of tunable geometric features, such as the aforementioned fillets and tuning stubs, which act to disproportionately adjust the scattering parameters of the two modes to obtain equivalent transmission properties. The viable range of parameters $(\phi$ and $\theta)$ are obtained by setting $(\epsilon=0)$, for a matched circuit, and solving for a real phase delay $(\operatorname{Im}(\psi)=0)$,

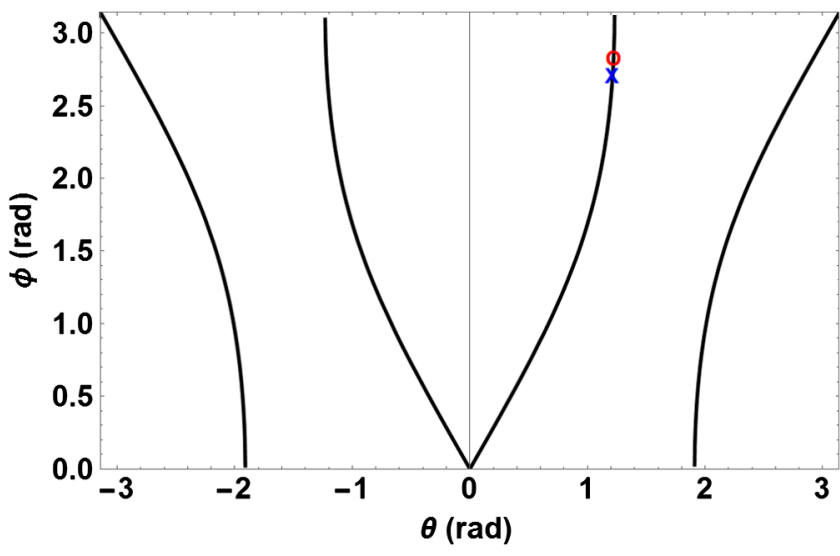

FIG. 5. A range of valid phase delays $\phi$ (black-line) based on the magnitude of the reflection $\theta$ at the cylindrical waveguide for network-BC with annotations denoting operating points of the polarizer for mode-1 (blue-X) and mode-2 (red-O) as measured in HFSS. 
$\phi(\theta)=-\arccos \left\{\frac{-1+5 \epsilon^{2}-3 \cos (2 \theta)-\epsilon^{2} \cos (2 \theta)}{4\left[-\epsilon-\cos (\theta)+\epsilon^{2} \cos (\theta)+\epsilon \cos (2 \theta)\right]}\right\}$.

The solution to Eq. (14) is plotted in Fig. 5.

\section{Network $A$}

The parameters of Network-A, from Fig. 6, are assumed to be an ideal losses matrix with 32 unknown terms which may be immediately simplified by targeting a matched network which, unlike a three port network, valid in a 4-port system. Furthermore, the coupling between the $T E_{10}^{\square}\left(S_{3 A: 1}\right)$ and $T E_{20}^{\square}\left(S_{3 A: 2}\right)$ is zero, eliminating the terms $S_{3 A: 1(3 A: 2)}$ and $S_{3 A: 2(3 A: 1)}$ and producing the matrix,

$S_{A}=\left(\begin{array}{cccc}0 & 0 & S_{1(3 A: 1)} & S_{1(3 A: 2)} \\ 0 & 0 & S_{2(3 A: 1)} & S_{2(3 A: 2)} \\ S_{1(3 A: 1)} & S_{2(3 A: 1)} & 0 & 0 \\ S_{1(3 A: 2)} & S_{2(3 A: 2)} & 0 & 0\end{array}\right)=\left(\begin{array}{cc}S_{w w} & S_{w x} \\ S_{x w} & S_{x x}\end{array}\right)$.

Provided that network-A has no coupling between the two modes (3A:1 and 3A:2), and contains two fold symmetry with respect to port- 1 and port-2, the magnitudes of the remaining scatter parameters must be equal $\left(\left|S_{1(3 A: 1)}\right|=\left|S_{2(3 A: 1)}\right|=\left|S_{1,(3 A: 2)}\right|=\left|S_{2(3 A: 2)}\right|\right)$. The phase

\section{Network-A}

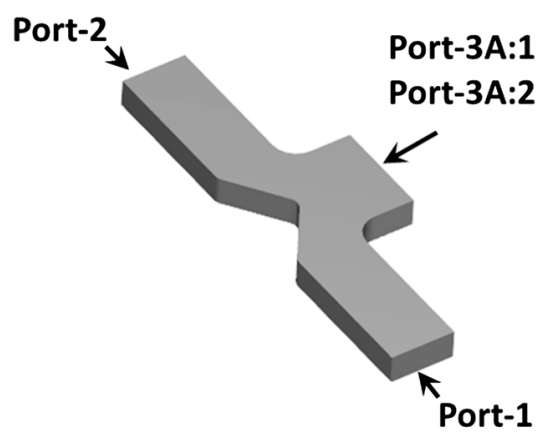

Network-BC

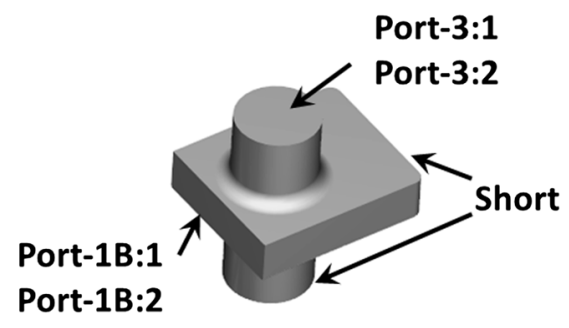

FIG. 6. Subnetworks A and BC used for the second cascade of the polarizer model with labeled port assignments. of these scattering parameters, denoted as $\left[\psi_{1(3 A: 1)}\right.$, $\left.\psi_{1(3 A: 2)}, \psi_{2(3 A: 1)}, \psi_{2(3 A: 2)}\right]$, must also preserve the unitary matrix resulting in the condition described by,

$$
\psi_{1(3 A: 1)}-\psi_{2(3 A: 1)}+\psi_{1(3 A: 2)}-\psi_{2(3 A: 2)}= \pm \pi .
$$

This phase-condition is inherently satisfied within the polarizer by the orientation of the plane of symmetry entering the overmoded rectangular waveguide. Assuming the reference phase for port-1 and port-2 are equal, the mirrored spatial orientation of each input port allows for the excitation of an in-phase $T E_{10}^{\square}$ mode but opposite polarities of the $T E_{20}^{\square}$ mode at ports-3A:1 and $3 A: 2$. This relative phase difference allows for each port to excite the opposite sense-polarization within the cylindrical waveguide while maintaining the desired directivity between the rectangular waveguide ports. Equation (15) can therefore be expressed using the relation $\left[\psi_{1(3 A: 1)}=\right.$ $\left.\psi_{2(3 A: 1)}\right]$ and $\left[\psi_{1(3 A: 2)}=\psi_{2(3 A: 2)}+\pi\right]$,

$$
\begin{aligned}
S_{w w} & =\left(\begin{array}{cc}
0 & 0 \\
0 & 0
\end{array}\right) \\
S_{x w} & =\left(\begin{array}{cc}
\frac{\sqrt{2}}{2} e^{i \psi_{1(3 A: 1)}} & \frac{\sqrt{2}}{2} e^{i \psi_{1(3 A: 2)}} \\
\frac{\sqrt{2}}{2} e^{i \psi_{1(3 A: 1)}} & \frac{\sqrt{2}}{2} e^{i \psi_{1(3 A: 2)}+\pi}
\end{array}\right) \\
S_{w x} & =\left(\begin{array}{cc}
\frac{\sqrt{2}}{2} e^{i \psi_{1(3 A: 1)}} & \frac{\sqrt{2}}{2} e^{i \psi_{1(3 A: 1)}} \\
\frac{\sqrt{2}}{2} e^{i \psi_{1(3 A: 2)}} & \frac{\sqrt{2}}{2} e^{i \psi_{1(3 A: 2)}+\pi}
\end{array}\right) \\
S_{x x} & =\left(\begin{array}{cc}
0 & 0 \\
0 & 0
\end{array}\right) .
\end{aligned}
$$

\section{The full network-ABC}

Network-BC shown in Fig. 6, having already been reduced to a 4-mode matrix in Eq. (11), is also decomposed into submatrices based on the interconnectivity with network-A,

$$
\begin{aligned}
S_{B C} & =\left(\begin{array}{cc}
S_{y y} & S_{y z} \\
S_{z y} & S_{z z}
\end{array}\right) \\
S_{y y} & =\left(\begin{array}{cc}
\frac{A_{1}-A_{1}^{2} Z_{1}+B_{1}^{2} Z_{1}}{1-A_{1} Z_{1}} & 0 \\
0 & \frac{A_{2}-A_{2}^{2} Z_{2}+B_{2}^{2} Z_{2}}{1-A_{2} Z_{2}}
\end{array}\right) \\
S_{z y}=\left[S_{y z}\right]^{T}= & \left(\begin{array}{cc}
\frac{C_{1}\left(A_{1} Z_{1}-B_{1} Z_{1}-1\right)}{A_{1} Z_{1}-1} & 0 \\
0 & \frac{C_{2}\left(A_{2} Z_{2}-B_{2} Z_{2}-1\right)}{A_{2} Z_{2}-1}
\end{array}\right) \\
S_{z z} & =\left(\begin{array}{cc}
\frac{D_{1}+C_{1}^{2} Z_{1}-A_{1} D_{1} Z_{1}}{1-A_{1} Z_{1}} & 0 \\
0 & \frac{D_{2}+C_{2}^{2} Z_{2}-A_{2} D_{2} Z_{2}}{1-A_{2} Z_{2}}
\end{array}\right) .
\end{aligned}
$$


$S(1,3: 2) \ldots S(2,1) \quad \circ S(1,3: 1)$. Relative Phase $(3: 1-3: 2)(\mathrm{rad})$

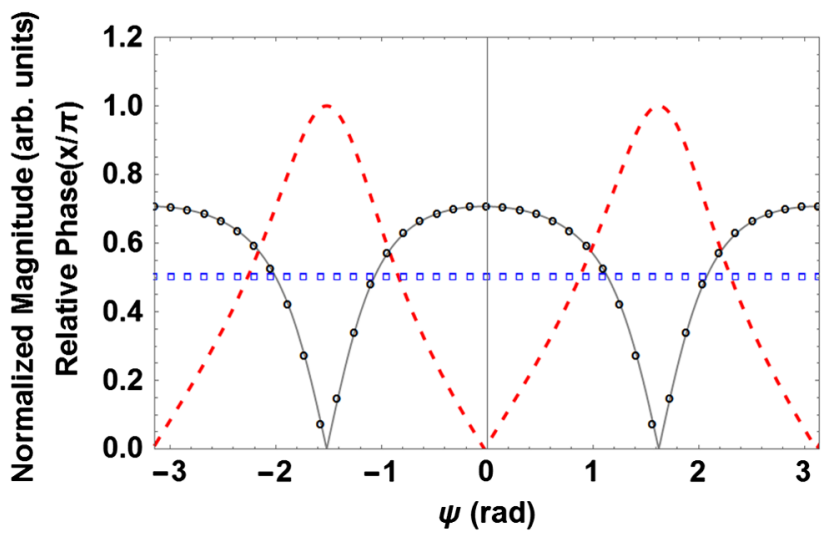

FIG. 7. S-parameters of the ideal circuit model with $S_{1(3: 1)}$ (black-o), $S_{1(3: 2)}$ (gray-line), $S_{21}$ (dashed-red), and the relative phase between each $T E_{11}^{O}$ mode (blue-squares), $S_{11}$ (not shown) is zero by design for this analytic model.

The final cascade operation removes the interconnected ports $(1 \mathrm{~B}: 1,1 \mathrm{~B}: 2$ and $3 \mathrm{~A}: 1,3 \mathrm{~A}: 2)$ to generate a 4-port network consisting of the two, single-mode, rectangular waveguide ports (port-1 and port-2) as well as the two $T E_{11}^{O}$ modes of the cylindrical waveguide (port-3:1 and port$3: 2$ ). Since it has already been assumed that both circuits are perfectly matched, and network-BC was tuned such that $\phi_{1}=\phi_{2}$, quadrature is preserved simply by incorporating a $\pi / 2$ phase delay between $\psi_{1(3 A: 1)}$ and $\psi_{1(3 A: 2)}$. Figure 7 illustrates the ideal scattering parameters of network-ABC as a function of $\left(\psi_{1}\right)$, where $\theta \sim 1.23$ and $\theta \sim 2.9$ as determined by Eq. (14). This configuration demonstrates transmission characteristics which are identical to that of a 180-degree $3 \mathrm{~dB}$ hybrid coupler,

$$
S_{T}=\left(\begin{array}{cccc}
0 & 0 & \frac{i}{\sqrt{2}} & \frac{1}{\sqrt{2}} \\
0 & 0 & \frac{i}{\sqrt{2}} & \frac{1}{\sqrt{2}} \\
\frac{i}{\sqrt{2}} & \frac{i}{\sqrt{2}} & 0 & 0 \\
\frac{1}{\sqrt{2}} & \frac{1}{\sqrt{2}} & 0 & 0
\end{array}\right)
$$

only for values of $\psi=n \cdot \pi$ for $(n=0,1,2 \ldots \infty)$.

\section{B. Finite element comparison to cascade model}

Numerical design of this device was performed in the finite element code HFSSV.15 using a driven-modal (network analysis) solution [15]. Each rectangular waveguide port was terminated using a single-mode, matched, waveport and the cylindrical waveguide was assigned a 2 -mode wave-port with a $\pi / 2$ radians offset. Similar to the analytic model, the geometry was split into segments and each mode of network-B was isolated by assigning perfect-E and perfect-H boundaries along the $\mathrm{H}$-plane axis of symmetry. The initial design goal of network-B was to minimize the reflection terms (S11, S22, S33) thus limiting viable solutions to $(\pi / 4<\theta<\pi / 2)$ and $(1.15<\phi<3)$ by Eq. (14). The components defined by networks-A and $C$ were evaluated as part of the full device shown in Fig. 1 and were numerically optimized to obtain a matched structure that both maintained quadrature for the $T E_{11}^{O}$ modes and isolation between ports-1 and 2. This numerical study of the full geometry utilized all of the geometric parameters of Fig. 1 including the cylindrical tuning stub, length of the overmoded rectangular waveguide, as well as additional the fillet radii at each waveguide juncture. The S-parameters of the final design, shown in Table I, closely matched to the ideal polarizer parameters shown in Eq. (19). The analytic parameters, shown in Table II, were backed out of the final design, post optimization, to assess the agreement of our simplified model with the numerical High Frequency Structure Simulator (HFSS) solution.

Table II shows that $\theta_{1}$ and $\theta_{2}$ are at the asymptotic limit for minimizing the mismatch between the cylindrical and rectangular waveguide. Additionally, the phase delay of each mode, $\phi_{1}$ and $\phi_{2}$, both fall within the predicted range of values from Eq. (14). In the HFSS model, quadrature is achieved by tuning $\psi_{1}$ and $\psi_{2}$ to have a $\pi / 2$ radian difference in network-C; as opposed to our analytic model which equivalently assumed the offset existed between $\psi_{3 A: 1}$ and $\psi_{3 A: 2}$ from network-A. The numerically optimized design deviates from the ideal model only when comparing the reflections from networks-A and BC. Preserving structural simplicity and compactness in subnetwork-A prevented the simultaneous matching of the $T E_{10}^{\square}$ and $T E_{20}^{\square}$ modes at ports-3A:1 and 3A:2. The resultant mismatch, $S_{3: 1,3: 1}=-10 \mathrm{~dB}$ and $S_{3: 2,3: 2}=-13 \mathrm{~dB}$, in subnetwork-A induced a proportional mismatch in network-BC to compensate for the reflection. This minor deviation from the ideal design had little impact on overall performance.

TABLE I. Scattering matrix of rf polarizer from HFSS.

\begin{tabular}{llccc}
\hline \hline $\mathrm{dB}(\mathrm{deg})$ & $\mathrm{S}: 1: 1$ & $\mathrm{~S}: 2: 1$ & $\mathrm{~S}: 3: 1$ & $\mathrm{~S}: 3: 2$ \\
\hline $\mathrm{S}: 1: 1$ & $-37.7(-105)$ & $-43.4(23)$ & $-3.01(60)$ & $-3.01(-30)$ \\
$\mathrm{S}: 2: 1$ & $-43.4(23)$ & $-37.7(-105)$ & $-3.01(-120)$ & $-3.01(-30)$ \\
$\mathrm{S}: 3: 1$ & $-3.01(60)$ & $-3.01(-120)$ & $-34.9(63)$ & $-99.2(35)$ \\
$\mathrm{S}: 3: 2$ & $-3.01(-30)$ & $-3.01(-30)$ & $-99.5(35)$ & $-39.8(-166)$ \\
\hline \hline
\end{tabular}


TABLE II. Variables derived from subnetwork analysis in HFSS.

\begin{tabular}{lccll}
\hline \hline Mode $(\mathrm{n})$ & $\theta_{n}$ & $\phi_{n}$ & $\psi_{n}$ & $\epsilon_{n}$ \\
\hline 1 & 1.215 & 2.70 & 0.524 & 0.3 \\
2 & 1.228 & 2.82 & 2.1 & 0.22 \\
\hline \hline
\end{tabular}

\section{APPLICATION TO PULSE COMPRESSION}

The passive pulse compression networks currently employed by the SLAC linac are S-band systems, based on the SLED-I archetype, which use two over coupled cylindrical cavities. The output of these structures feed an amplified pulse to the accelerating structure via a 4-port, $3-\mathrm{dB}$, hybrid coupler to provide isolation to the rf source. The compact rf polarizer described in Sec. II vastly reduces the complexity of this rf network as only a single cavity is required to yield the same passive functionality. In this configuration, the resonant cavity must also be capable of supporting two modes in quadrature to maintain proper coupling to the polarized mode. The response function of the SLED cavity is described by the sum of the reflected $\left(E_{i}\right)$ and emitted $\left(E_{e}\right)$ signal from the resonant structure with amplitude given by, [16].

$$
E_{\text {out }}=E_{e}-E_{i} \text {. }
$$

Following the conservation of energy in the resonant system,

$$
d U / d t=P_{i}-P_{o}-P_{L},
$$

the power inside the cavity can be described in terms of the input rf signal $\left(P_{i}\right)$, the output rf signal $\left(P_{o}\right)$ and the $\mathrm{rf}$ resistive losses $\left(P_{L}\right)$. The losses depicted in Eq. (21) can be readily expressed in terms of the resonator characteristics, ( $Q_{0}$ - unloaded quality factor and $\beta$-coupling factor), where $P_{L} \sim \frac{E_{e}^{2}}{\beta}$ and $U=\frac{Q_{0}}{\omega} P_{L}$ such that $\frac{d U}{d t}=\frac{2 Q_{0}}{\omega \beta} E_{e} \frac{d E_{e}}{d t}$. Plugging these parameters into Eq. (21), provides the first order differential,

$$
\tau \frac{d E_{e}}{d t}+E_{e}=\frac{2 \beta}{1+\beta} E_{i},
$$

where the cavity fill time is defined as $\tau=\frac{2 Q_{L}}{\omega}$ and $Q_{L}$ is the loaded quality factor. The output signal $E_{\text {out }}$ is calculated by solving Eq. (22) using a piecewise input signal to represent initial energy storage $\left(E_{i}=E_{0}\right.$, from $t=T_{1}$ to $\left.t=T_{2}\right)$, phase reversal and power dissipation $\left(E_{i}=-E_{0}\right.$, from $t=T_{2}$ to $\left.t=T_{3}\right)$, and turn off $\left(E_{i}=0\right.$, where $\left.t>T_{3}\right)$.

Alternatively, the output waveform of the SLED cavity can be solved for by a convolution of the input signal and cavity response function (scattering parameters). A simple

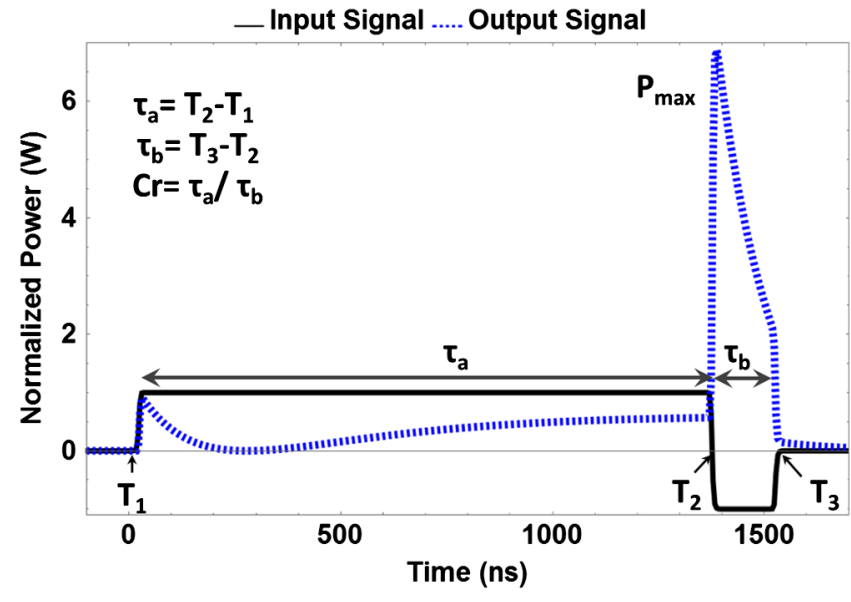

FIG. 8. Numerically derived output power (dashed:blue) using the convolution of an RLC resonator model and standard input pulse (black) for passive pulse compression cavities.

model for the SLED scattering matrix can be derived by solving for the reflection coefficient $(\Gamma)$ of a single port resonant circuit,

$$
\Gamma=S 11=\frac{Z-Z 0}{Z+Z 0}=\frac{1}{\beta} \frac{\left(1+j Q_{0} \delta\right)-1}{\left(1+j Q_{0} \delta\right)+1},
$$

where $\delta=\frac{\omega}{\omega_{0}}-\frac{\omega_{0}}{\omega}$ and $\omega_{0}$ is the resonant frequency of the SLED cavity. Figure 8 , derived via convolution of our simplified circuit model from Eq. (23) with a $11.424 \mathrm{GHz}$ square waveform, illustrates the characteristic SLED output signal once the phase is reversed at $\left(t=T_{2}\right)$. This particular pulse was modeled using a $Q_{0}=1 \times 10^{5}$, a coupling coefficient $(\beta)$ of 9 , a pulse length $\left(\tau_{b}\right)$ of $150 \mathrm{~ns}$, and a compression ratio $(\mathrm{Cr})$ of 9 (where $\mathrm{Cr}$ is defined as $\frac{\tau_{a}}{\tau_{b}}$ ).

\section{A. Spherical cavity}

The current compact, single-cavity, SLED system under investigation uses a spherical resonator [7]. Spherical $T E_{n m \rho}$ modes (Fig. 9B) whose electric vector potential is defined by the mode number $(n, m, \rho)$, the root of the spherical Bessel equation $\left(u_{n \rho}\right)$, and radius (a),
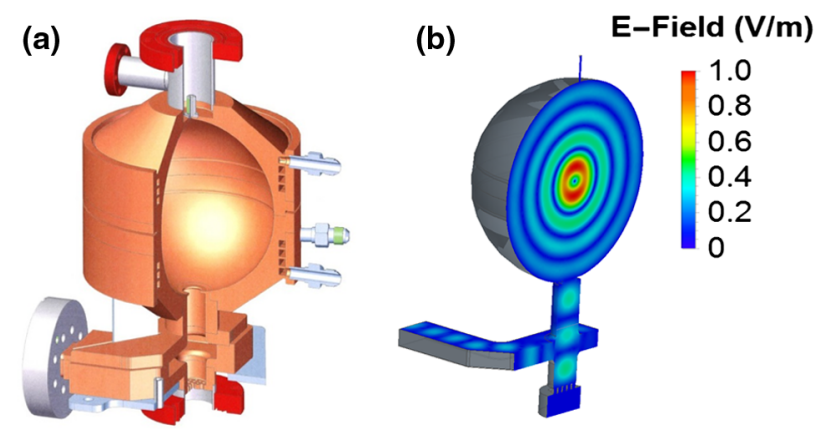

FIG. 9. (a) Cutaway CAD drawing of the spherical cavity used for pulse compression with RF polarizer, and (b) contour plot $T E_{141}$ mode in the spherical cavity calculated using HFSS. 


$$
\mathbf{F r}_{n m \rho}=J_{n}\left(u_{n \rho} \frac{r}{a}\right) P_{n}^{m}[\cos (\theta)] \cos (m \phi),
$$

are ideal for this application due to both their high- $Q$ $\left(Q_{0}=\frac{a}{\delta}\right.$, where $\delta$ is the rf skin depth) and number of available degenerate modes. These orthogonal modes are only mildly separated by the introduction of a cylindrical coupler to the base of the device shown in Fig. 9(A) and, if properly excited, will preserve the state of quadrature demanded by the polarizer. The initial design of the spherical resonator utilized the $T E_{m 41}$ mode set in the realm of $11.424 \mathrm{GHz}$, dictating a device radius of approximately $5.875 \mathrm{~cm}$ with consideration of external loading. This set of modes defines three degenerate eigensolutions, the $T E_{114 e}, T E_{114 o}$, and $T E_{014}$ mode(s). Unperturbed, each of these modes share the same resonant frequency,

$$
f_{0}=\frac{u_{n \rho}}{2 \pi a \sqrt{\epsilon \mu}},
$$

where $\left(u_{n \rho}\right)$ is the zero of the spherical Bessel equation for the $T E_{m n \rho}$ mode.

\section{B. Cold test of polarizer}

The body of the rf polarizer, shown in Fig. 10(A), was milled from bulk oxygen free electronics (OFE) grade

(a)

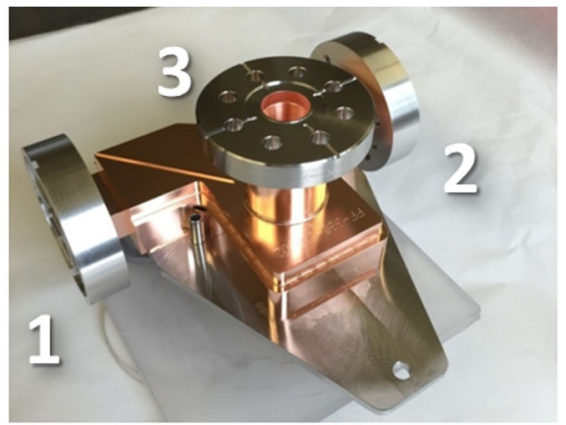

(b)

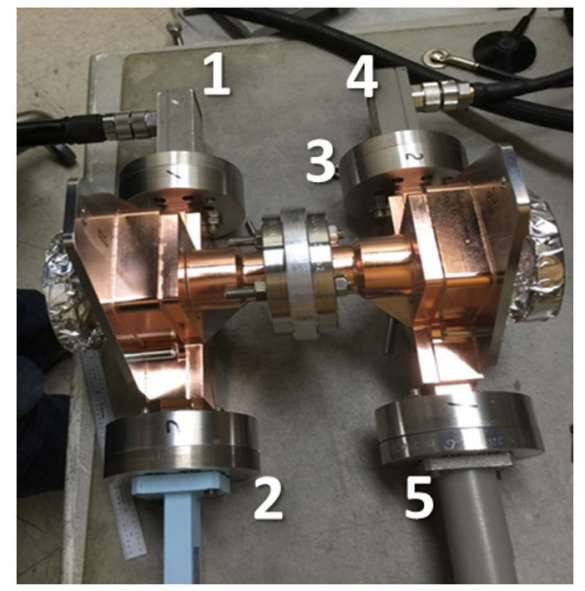

FIG. 10. (a) Photograph of the rf polarizer with no attachments, and (b) photograph of two rf polarizers connected via the cylindrical waveguide. copper in two parts and brazed together to form the body of the device. Each WR 90 port was terminated with male SLAC crush seal waveguide flanges and a custom flange was designed to accommodate a nonstandard $1.95 \mathrm{~cm}$ ID of the cylindrical waveguide. A port for vacuum pumping was included below the "shorted" plane of the cylindrical waveguide located on the bottom of the device but was electrically cutoff to $11.424 \mathrm{GHz}$.

Cold tests were performed with three separate configurations:

A. using an electrical short installed over the cylindrical waveguide (port-3),

B. with an identical rf polarizer connected through a cylindrical spacer, as shown in Fig. 10(B), and

C. with the spherical cavity installed at the cylindrical waveguide port.

In configuration-A power incident from port-1 will reflect off of the cylindrical short circuit therein reversing the direction of the polarized wave. The reflected signal will couple to a $T E_{10}^{\square}$ and $T E_{20}^{\square}$ that are of opposite phase of the incoming signal thus providing transmission to port-2 and isolation from port-1. Configuration- $\mathrm{B}$ is designed to preserve the directionality of the polarized mode between the two rf polarizers and provide isolation to ports 2 and 5 . Both configurations used APC-7 to WR 90 (instrument) adapters, equipped with a zero length instrument to vacuum (crush seal) flange on each WR 90 waveguide. The remaining ports in configuration (B), ports-2 and 5, were terminated with matched loads. All measurements were performed with an Agilent 5242A pulsed network analyzer and the system was calibrated using a Transmission-LineThru (TRL) kit for WR 90.

In the first set of measurements, configuration-A, the frequency bandwidth was set to $100 \mathrm{MHz}$ spanning from $11.374 \mathrm{GHz}$ to $11.474 \mathrm{GHz}$ with 801 points. The scattering parameters in Fig. 11 A demonstrate excellent agreement with analytic design and simulation, showing a reflection of approximately $-46 \mathrm{~dB}$ at the operating frequency and $-40 \mathrm{~dB}$ over the viable bandwidth of the device. Transmission through the structure from port-1 to port-2 (S21), from Fig. 11(B) varied between -0.075 and $-0.090 \mathrm{~dB}$ and showed no indication of trapped modes. Similarly, configuration-B showed exceptional transmission from 11.36 to $11.48 \mathrm{GHz}$, with $S_{41}$ was on the order of $-0.05 \mathrm{~dB}$ and the reflected signal as low as $-54 \mathrm{~dB}$ at the operating frequency. Slight discrepancies in transmitted power can be attributed to the added resistivity of the aluminum disk used as a short in configuration-A.

The addition of the spherical resonant cavity in configuration- $\mathrm{C}$ produced a reflected power signal which was measured at port-2 and plotted in Fig. 12. The measurements for this configuration were made over a $40 \mathrm{MHz}$ bandwidth (11.40-11.44 GHz) and identified two resonances at $11.4215 \mathrm{GHz}\left(T E_{114}\right)$ and $11.427 \mathrm{GHz}\left(T E_{014}\right)$. These modes were identified by correlating the cold test 

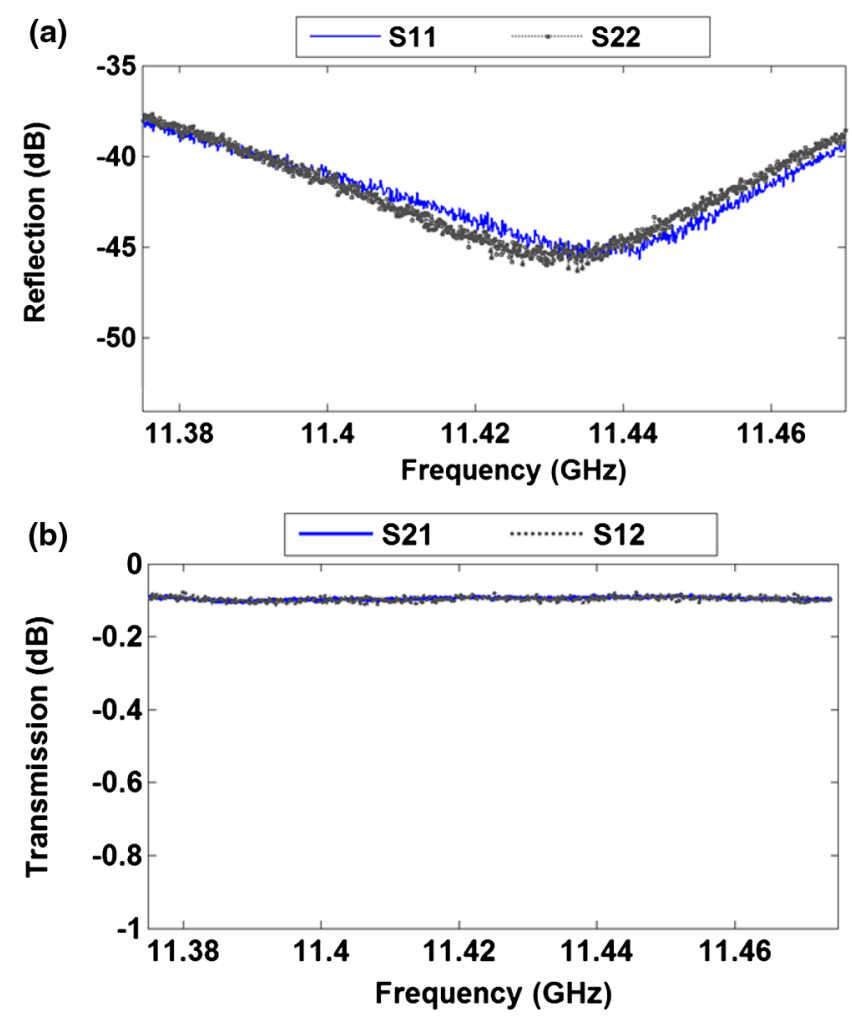

FIG. 11. Cold test results of configuration:A, measured with an Agilent N5242A PNA-x showing (a) the reflection characteristics S11 and S22, and (b) the transmission properties S21 and S12.

data with similar measurements simulated in HFSS. These modes, which are degenerate for a perfectly conducting spherical resonator, experienced disproportionate coupling with the output waveguide which loaded the $T E_{114}$ mode significantly more than the $T E_{014}$. Based on the excellent return loss measured in configurations- $\mathrm{A}$ and $\mathrm{B}$ the two port measurement $\mathrm{S} 21$ could be approximated as a, single port, RLC circuit model which was used in the calculation of quality factor and coupling shown in Table III.

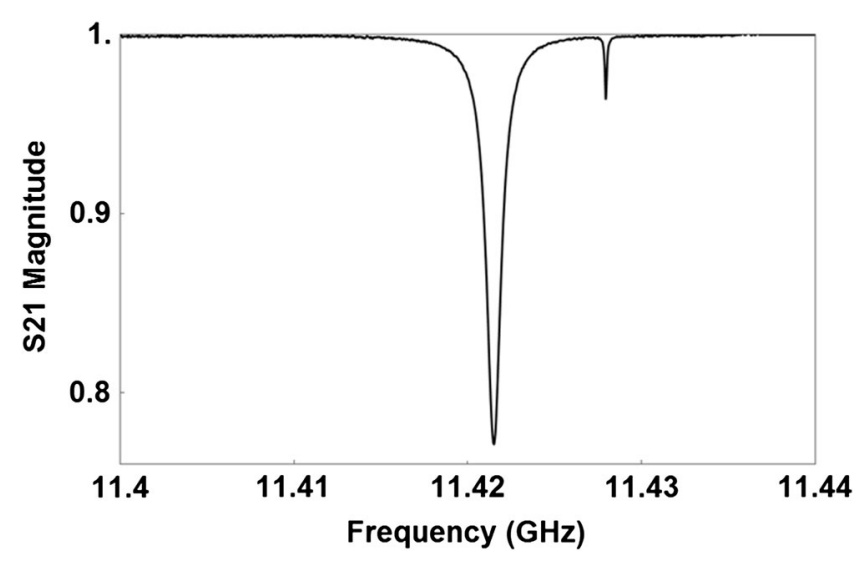

FIG. 12. Cold test results of configuration-C measured with an Agilent N5242A PNA-X showing the transmission properties S21 of the network.
TABLE III. Measured resonator characteristics of spherical cavity ( $T E_{114}$ mode).

\begin{tabular}{lccc}
\hline \hline$Q_{0}$ & $Q_{L}$ & $\beta$ & $f_{0}$ \\
\hline $9.23 \times 10^{4}$ & $1.06 \times 10^{4}$ & 7.69 & $11.4215 \mathrm{GHz}$ \\
\hline \hline
\end{tabular}

\section{High power test}

The SLED cavity with polarizer was installed at the Klystron Test Laboratory at SLAC and driven using an XL-4 klystron as shown in Fig. 13(A). Following the experimental setup from Fig. 13(B), the $1.5 \mu$ s low level rf (LLrf) pulse $(3 \mathrm{dBm})$ from (1) is modified to include a reversal of phase after $1.335 \mu$ s via an Agilent arbitrary function generator (2). The waveform is amplified downstream by both a low power $20 \mathrm{~dB}$ gain amplifier (3) as well as a traveling wave tube (4) to bring the power level to $\sim 1 \times 10^{5} \mathrm{dBm}$ before being passed to the XL-4 klystron (5). The high power, multi-megawatt, signal at the output of (5) is sampled by (6a-forward) and (6b-reverse) before reaching the polarizer (7). The polarizer (7) and SLED resonator (8) were assembled in the same manner as configuration (C) from cold tests, which minimized reflection back to (5). The output signal from (8) was sampled with $-75 \mathrm{~dB}$ of attenuation via the peak power meters (9a)

(a)
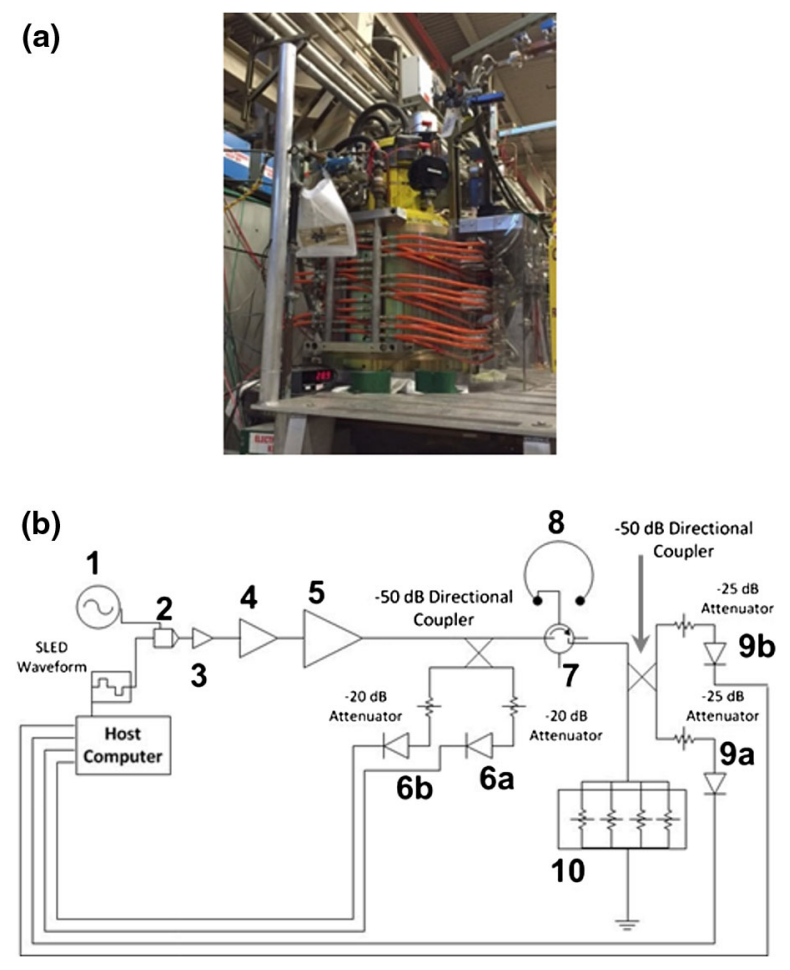

FIG. 13. (a) Photograph of the XL-4 klystron used to test the SLED cavity and (b) a circuit diagram of the experimental setup: (1) local oscillator, (2) arbitrary function generator (AFG), (3) 20db amplifier, (4) traveling wave tube, (5) SLAC XL4 klystron, (6a-6b) peak power meters before SLED, (7) polarizer, (8) SLED, (9a-9b) peak power meters after SLED, and (10) high power rf load. 


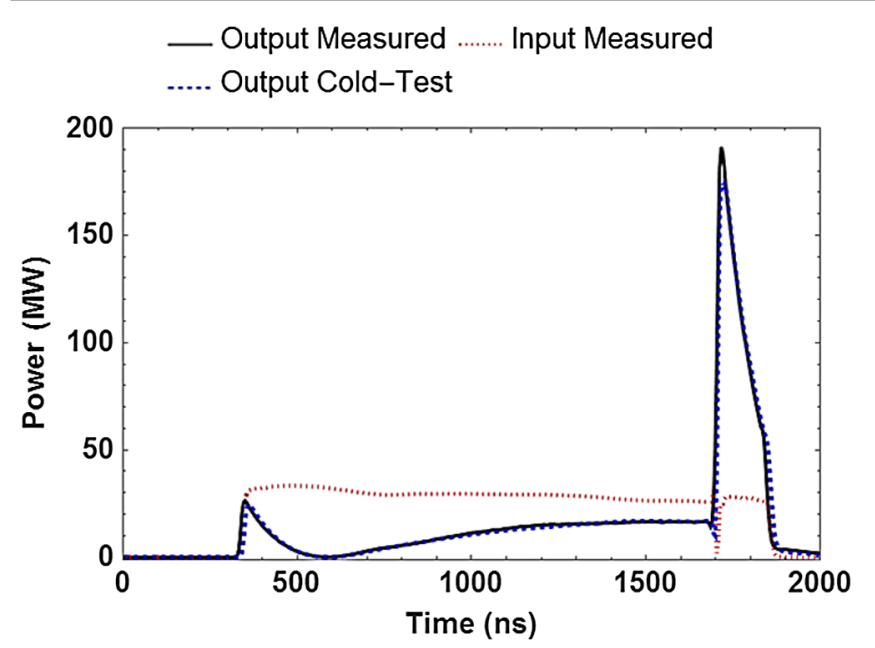

FIG. 14. A chart showing the measured input pulse envelope (dotted:Red), the measured output pulse (black), and the predicted output pulse (dashed:Blue) based on the cold test measurement.

and (9b) before the power is dissipated into a high power $\mathrm{rf}$ load (10). Both the SLED and the rf network were conditioned by gradually ramping the average input power from 0 to $32 \mathrm{MW}$ with a constant compression ratio of 8.09 at a repetition rate of $60 \mathrm{~Hz}$. During this process breakdowns were observed with gas burst within the rf waveguide but the SLED system itself demonstrated no propensity for high power breakdown. Peak SLED output powers were observed up to $207 \mathrm{MW}$ with an average power of $132 \mathrm{MW}$ over the duration of the $150 \mathrm{~ns}$ signal. The waveforms in Fig. 14 represents both the experimental input (dotted:light-gray) and output (black) as well as a cold-test output (dashed:gray) (generated by performing a convolution of the cold-test scattering parameters and the experimental input pulse).

\section{Conclusion}

This article describes the design process of the rf polarizer, its microwave characterization, and the high power test as a part of the multi-MW rf pulse compressor. The rf polarizer is a versatile device could be used for a variety of high power microwave components such as phase-shifters, circulators, and high power rf loads. The device was numerically analyzed as a set of subnetworks. Each subnetwork was optimized to arrive at the final design. Four high power devices were manufactured at SLAC. Cold tests of two back-to-back polarizers demonstrated isolation and return loss of $-40 \mathrm{~dB}$ and transmission of $-0.05 \mathrm{~dB}$ over the $100 \mathrm{MHz}$ bandwidth. One of the devices was used as part of a novel rf pulse compressor. This rf pulse compressor was tested at high power at the Klystron Test Laboratory at SLAC. The test demonstrated the polarizer's ability to handle rf power in excess of 200 MW. Future work will continue to expand the application of the rf polarizer in high power environments.

\section{ACKNOWLEDGMENTS}

This work was supported by DOE Contract No. DEAC02-76SF00515. Special thanks to Jim Lewandowski, John Eichner, and Joseph Olszewski for their contributions and expertise in operating the experimental setup at the SLAC Klystron Test Facility. We also thank Gordan Bowden and Andy Haase for their contributions to the mechanical design and assembly of both the rf polarizer and SLED cavity.

[1] E. Schamiloglu, High power microwave sources and applications, Microwave Symposium Digest, 2004 IEEE MTT-S International (2004), Vol. 2, pp. 1001-1004.

[2] High Power Microwave Sources and Technologies, edited by R. J. Barker and E. Schamiloglu (J. Wiley Sons, New York, 2001).

[3] Z. D. Farkas, H. A. Hogg, G. A. Loew, and P. B. Wilson, SLED: A method of doubling SLAC's energy, in Proceedings of 9th International Conference on High Energy Accelerators (SLAC, 1974), p. 576.

[4] P. B. Wilson, Z. D. Farkas, and R. D. Ruth, in Proceedings of the 22nd 1990 LINAC Conference, PAC-2007, Albuquerque, NM (IEEE, New York, 2007).

[5] D. Farkas, Binary peak power multiplier and its application to linear accelerator design, IEEE Trans. Microwave Theory Tech. 34, 1036 (1986).

[6] S. Tantawi, U.S. Patent Application 14/641,938, 2015.

[7] J. Wang, S. Tantawi, and X. Chen, Super-compact SLED system used in the LCLS diagnostic system, in Proceedings, 27th Linear Accelerator Conference, LINAC 2014, Geneva, Switzerland (JACoW, 2014).

[8] W. R. Fowkes, E. N. Jongewaard, R. J. Loewen, S. G. Tantawi, and A. E. Vlieks, in Proceedings of the Particle Accelerator Conference, Vancouver, BC, Canada, 1997 (IEEE, New York, 1997).

[9] C. Chang, L. Guo, S. Tantawi, Y. Liu, J. Li, C. Chen, and W. Huang, A new compact high-power microwave phase shifter, IEEE Trans. Microwave Theory Tech. 63, 1875 (2015).

[10] C. Chang, S. Tantawi, S. Church, J. Neilson, and P. V. Larkoski, Novel compact waveguide dual circular polarizer, Prog. Electromagn. Res. 136, 1 (2013).

[11] T. Kitsuregawa, Advanced Technology in Satellite Communication Antennas: Electrical and Mechanical Design (Artech House, Norwood, MA, 1990), p. 81.

[12] C. Nantista, Overmoded waveguide components for high power rf, AIP Conf. Proc. 691, 263 (2003).

[13] S. Tantawi, Multimoded reflective delay lines and their application to resonant delay line rf pulse compression systems, Phys. Rev. ST Accel. Beams 7, 032001 (2004).

[14] K. Gupta, R. Garg, and R. Chadha, NASA STI/Recon Technical Report No. A 82, 1981: 39449.

[15] ANSYS: Electronics Desktop (High Frequency Structure Simulator), http://www.ansys.com/Products/Electronics/ RF-and-Microwave, 2015.

[16] C. Nantista, Stanford University Report No. SLAC-R95455, UC-414, 1995. 